This item was submitted to Loughborough's Research Repository by the author.

Items in Figshare are protected by copyright, with all rights reserved, unless otherwise indicated.

\title{
Engineering design of artificial vascular junctions for 3D printing
}

PLEASE CITE THE PUBLISHED VERSION

http://dx.doi.org/10.1088/1758-5090/8/2/025018

PUBLISHER

(C) IOP Publishing

\section{VERSION}

AM (Accepted Manuscript)

\section{PUBLISHER STATEMENT}

This is an author-created, un-copyedited version of an article accepted for publication in Biofabrication. IOP Publishing Ltd is not responsible for any errors or omissions in this version of the manuscript or any version derived from it. The Version of Record is available online at http://dx.doi.org/10.1088/1758-5090/8/2/025018

\section{LICENCE}

CC BY-NC-ND 4.0

\section{REPOSITORY RECORD}

Han, Xiaoxiao, Richard Bibb, and Russell A. Harris. 2016. "Engineering Design of Artificial Vascular Junctions for 3D Printing". Loughborough University. https://hdl.handle.net/2134/21739. 


\section{Engineering Design of Artificial Vascular Junctions for 3D Printing}

2 Xiaoxiao Han ${ }^{\mathrm{a}, \mathrm{b} *}$, Richard Bibb ${ }^{\mathrm{b}}$, Russell Harris ${ }^{\mathrm{c}}$

3

$4{ }^{\mathrm{a}}$ Wolfson school of Mechanical, Electrical and Manufacturing Engineering, Loughborough University, 5 Loughborough. Leicestershire, LE11 3TU, UK

6 b Loughborough Design School, Loughborough University, Loughborough. Leicestershire, LE11 3TU, $7 \quad \mathrm{UK}$

$8 \quad{ }^{\mathrm{c}}$ School of Mechanical Engineering, University of Leeds, Leeds, LS2 9JT, UK.

9

10 *corresponding author, Email: x.han2@lboro.ac.uk, Tel: +44 (0)1509 227567

11 


\section{Abstract}

13 Vascular vessels, including arteries, veins and capillaries, are being printed using additive 14 manufacturing technologies, also known as 3D Printing. This paper demonstrates that it is important 15 to follow the vascular design by nature as close as possible when 3D printing artificial vascular 16 branches. In previous work, the authors developed an algorithm of computational geometry for 17 constructing smooth junctions for 3D printing. In this work, computational fluid dynamics (CFD) is used to compare the wall shear stress and blood velocity field for the junctions of different designs.

19 The CFD model can reproduce the expected wall shear stress at locations remote from the junction.

20 For large vessels such as veins, it is shown that ensuring the smoothness of the junction and using 21 smaller joining angles as observed in nature is very important to avoid high wall shear stress and 22 recirculation. The issue is however less significant for capillaries. Large joining angles make no 23 difference to the hemodynamic behavior, which is also consistent with the fact that most capillary 24 junctions have large joining angles. The combination of the CFD analysis and the junction 25 construction method form a complete design method for artificial vascular vessels that can be 3D 26 printed using additive manufacturing technologies.

27 Keywords: Vascular vessel design, Computational fluid dynamics (CFD), Additive Manufacturing, 3D printing 
31

$32 C_{\max }$ Non-dimensionalised maximum curvature of a bifurcation junction

$33 \quad \bar{V} \quad$ Non-dimensionalised branch Volume

$34 p_{\text {outlet }}$ Outlet pressure

$35 v_{\text {inlet }} \quad$ Inlet velocity

$36 x, y, z$ Cartesian coordinates

$37 \quad u_{d}$ Control parameters 


\section{Introduction}

3D Printing has made it possible for the first time to manufacture artificial blood vessels and their networks of any sophisticated geometry and connections. The printed vascular vessels can be used as grafts to treat inadequate blood flow or alongside organ transplantation (Edelman, 1999). In this case, polymers such as poly-propylene-glycol are printed layer by layer to construct a graft which will remain permanently inside the human body. Printed vascular networks can also be used in tissue engineering for regenerative medicine. In this case, biodegradable polymers such as poly-lactide-acid are printed to fabricate a scaffold for the endothelial cells to regenerate into blood vessels. Tissue engineering using a single graft offers potential advantages over conventional autologous or synthetic grafts (Edelman, 1999; Naito and Rathore, 2011). A major issue in organ tissue engineering is that the artificial organ may not develop adequate vascularisation for long-term survival (R.A.J. and O.C., 2002). An artificial micro-vascular network can provide nutrients and soluble growth factors to cells and tissues as well as acting as a scaffold for culturing endothelial cells for capillaries (Patrick Jr, 2000; Kannan et al., 2005; Kamel et al., 2013). The additive manufacturing technology offers the complete freedom to design the details of a vascular branch. Currently various research groups have successfully 3D printed and tested such vascular vessels (Wu et al., 2011; Miller et al., 2012; Kucukgul et al., 2013; Hoch et al., 2014; Kolesky et al., 2014). However, a general guidance on the design of the vascular branch is missing. There have been many published works studying the blood flow and wall shear stress in vascular networks using computational fluid dynamics (CFD). For example, the effect of branching angles on hemodynamics of bifurcations was investigated in great detail in (Friedman et al., 1983; Rabinovitz et al., 1987; Friedman and Seed, 1993; Edelman, 1999; Liu et al., 2015). It was concluded that the branching angle affects the distribution of the flow field in the branch. The flow will become fully developed downstream. The developed rate is determined by the Reynolds number. Local recirculation may occur inside a branch where the cells and nutrients aggregate. The wall shear stress (WSS) is a key hemodynamic indicator that affects the endothelial cell development (Ravensbergen et al., 1995; Ravensbergen et al., 1997; Kohler et al., 2001; Marshall et al., 2004). WSS outside a normal range between 1Pa to 7Pa (Papaioannou and Stefanadis, 2004) is considered harmful to vessel development and may lead to cardiovascular diseases (Kohler et al., 
2001; Caro, 2008; Coppola and Caro, 2008). However, the previous CFD studies were not aimed at providing guidance for the design of 3D printed vascular vessels. Issues related to 3D printing have not been studied. In the current practice, vascular networks are 3D printed without little understanding of their hemodynamics. "Artificial vascularised scaffolds for 3D-tissue regeneration (ArtiVasc 3D)” is a large project funded by the European Union's Seventh Framework Programme that aims to generate fully vascularised bioartificial skin. The work described in this paper is part of ArtiVasc 3D. In previous work, the authors developed an algorithm of computational geometry for the construction of vascular branch. The algorithm uses three main geometric parameters to control the branch geometry. They are branch angle, volume and maximum curvature at the apex. Three-dimensional models of vascular vessels are generated which can be translated into STL (Stereolithography) data file for 3D Printing (Gibson et al., 2010).

The purpose of this paper is to present general guidance on the design of vascular branches. CFD simulations are performed for artificial branches targeted for veins and micro-vascular vessels respectively. For each type of vascular branch, the effect of branch angle and local curvature on the blood flow behavior and WSS are studied. A laminate CFD model in commercial software, COMSOL, was used. Confidence in the CFD model was achieved by the fact that it reproduced the measured wall shear stresses away from the branch. For the large veins, it is shown that ensuring the smoothness of the junction and keeping a relatively small branch angle, as observed in human body, is very important to avoid high wall shear stress and recirculation. The issue is however less significant for capillaries. The branch angle in capillaries has little effect on the WSS, which can also be explained by the fact that capillaries in nature often have large branch angles. The combination of the CFD analysis and the junction construction algorithm form a complete design method for the vascular vessels.

\section{Design of vascular branches and their CFD models}

We consider artificial vascular vessels targeted for veins and micro-vascular vessels, respectively. Vascular branches are constructed using the algorithm described in (Han et al., 2015). Figure 1 shows the mid-sections of the smooth branches with three different joining angles of $45^{\circ}, 85^{\circ}$, and $125^{\circ}$. 


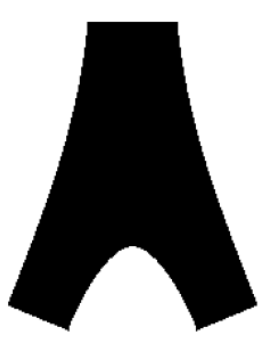

$45^{\circ}$

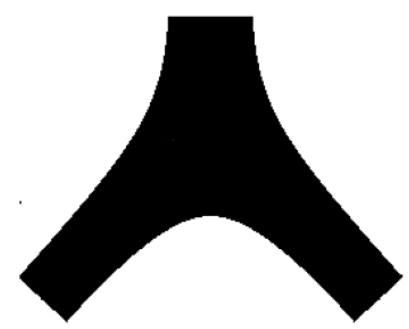

$85^{\circ}$

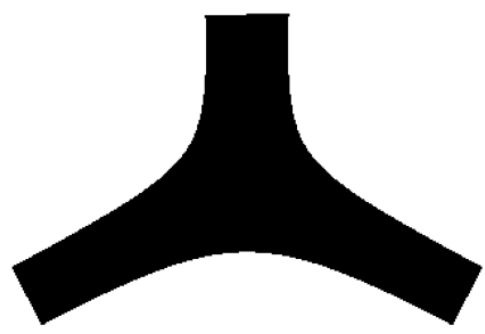

$125^{\circ}$
93

Figure 1. Mid-sections of the smooth branches with joining angles of $45^{\circ}, 85^{\circ}$, and $125^{\circ}$.

The branch is made by joining three circular tubes of two different diameters. For the veins, the diameters are 5mm and 3.87mm, respectively (Ravensbergen et al., 1995; Ravensbergen et al., 1997). The dimensions of the model were based on a morphological study of 85 human vertebra-basilar specimens. The ratio between the total cross-sectional area of the two inlet tubes and the crosssectional of the outlet tube is equal to 1.2 which is biologically realistic according to (Ravensbergen et al., 1995). For the micro-vascular vessels, the diameters are $0.05 \mathrm{~mm}$ and $0.0387 \mathrm{~mm}$, respectively. The shape of the junction profile shown in Figure 1 is taken as a Bezier curve [1]. Bezier curve uses a parameter $u_{d}$ to complete the definition of the curve, which is fully explained in [1]. Table 1 provides the values of $u_{d}$ for cases studied in this work. The different values of $u_{d}$ lead to different maximum curvatures of the junction, which are also provided in Table 1.

Table 1. Value of controlled variables for different cases

\begin{tabular}{|c|c|c|c|c|}
\hline $\begin{array}{l}\text { Parameters } \\
\text { Cases }\end{array}$ & $\begin{array}{c}\text { Branch } \\
\text { angle }\end{array}$ & $\bar{C}_{\max }$ & $\bar{V}$ & $u_{d}$ \\
\hline 1 & $125^{\circ}$ & 0.44 & 5.4 & 0.89 \\
\hline 2 & $85^{\circ}$ & 1.43 & 5.4 & 0.84 \\
\hline 3 & $45^{\circ}$ & 1.34 & 5.9 & 0.4 \\
\hline 4 & $45^{\circ}$ & 3.02 & 5.56 & 0.53 \\
\hline 5 & $45^{\circ}$ & 5.37 & 5.4 & 0.6 \\
\hline 6 & $45^{\circ}$ & 7.5 & 5.3 & 0.63 \\
\hline 7 & $45^{\circ}$ & 14 & 5.2 & 0.674 \\
\hline
\end{tabular}


107 The curvature is normalised by the diameter of the larger tube. Five different curvatures are used for 108 the branches with joining angle of $45^{\circ}$ to study the effect of different curvatures. As an extreme 109 example of unnatural design, vascular junctions are also constructed using rectangular tubes of two 110 different sizes. Bezier curves are not used to smooth the junction. The dimensions of the rectangular 111 tube are provided in figure 2.

112

Figure 2. Sharp branching dimensions and boundary conditions.

These dimensions are selected to ensure the circular and rectangular tubes have roughly the same cross section area. The junction is connected to two inlet tubes of $70 \mathrm{~mm}$ and one outlet tube of 200mm (Ravensbergen et al., 1995; Ravensbergen et al., 1997).

124 The model makes the following assumptions:

- The flow is laminar, incompressible and Newtonian;

- Only steady state flow is considered;

- The vessel walls are assumed as rigid with no-slip conditions;

When steady state and incompressible conditions are assumed, the Navier-Stoke equation can be written in terms of the velocity $\mathbf{u}$ and pressure $p$ :

$$
-\varepsilon \Delta \mathbf{u}+\mathbf{u} \cdot \nabla \mathbf{u}+\nabla p=\mathbf{f} \quad \text { in } \Omega
$$


132 where $\varepsilon$ is the viscosity and $\mathbf{f}$ is the body force per unit mass. In this study, $\varepsilon$ is set as $0.003 P a . s$ 133 following (Ravensbergen et al., 1995). As the boundary conditions, the inlet velocity at the two smaller tubes is set as $0.465 \mathrm{~m} / \mathrm{s}$ (Ravensbergen et al., 1997) while the outlet pressure at the larger tube is set as zero (Ravensbergen et al., 1997). Previous studies have shown that the flow behavior in the junction is insensitive to the actual value of the outlet pressure because of the large length of the outlet tube used in the model. The Reynolds number is 600 for veins and 0.06 for micro-vascular vessels both of which are within the normal range of human blood flow (Cliff, 1976). The CFD module of commercial software COMSOL ${ }^{\circledR}$ was used to solve the equations. A series of convergence studies were performed to ensure the finite element meshes are fine enough to produce the correct solutions. for bifurcation angle $63^{\circ}$.

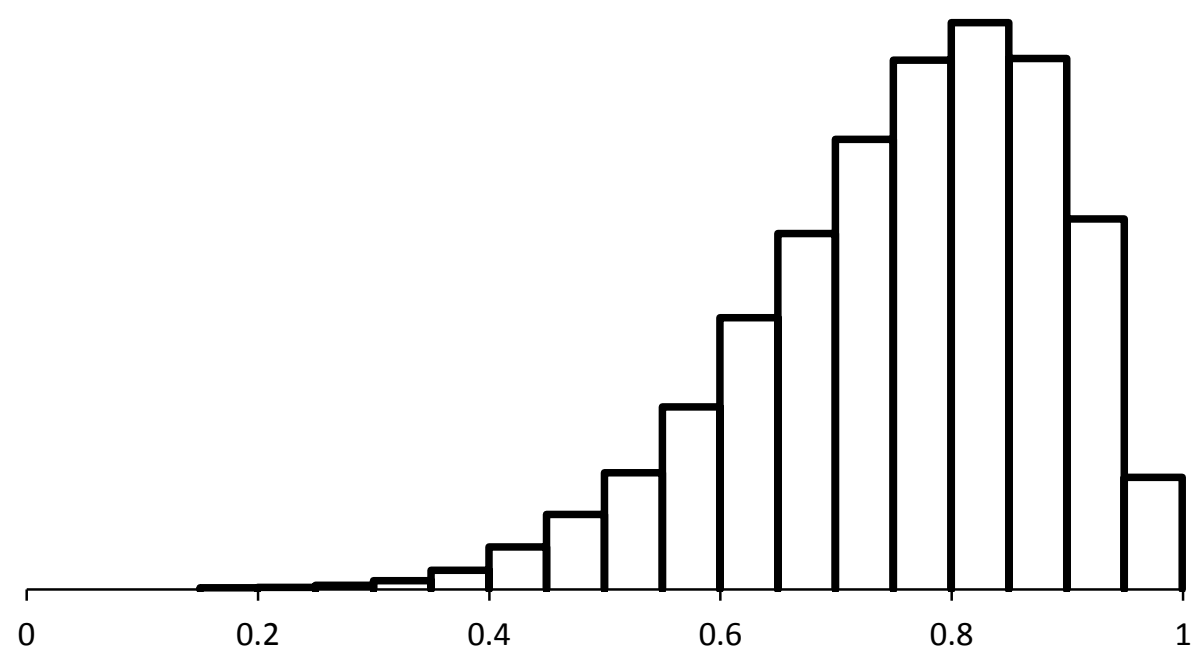

Figure 3. Histogram of mesh element quality distribution

Figure 3 shows a mesh element quality distribution histogram. The $\mathrm{x}$ axis in figure 3 is the quality indicator for mesh elements. For a best element, the indicator equals 1 and for other meshes, the indicator is in the range of $(0,1]$. Bad elements have smaller values. In statistics, the median value of this distribution is 0.55 ; the mean value of this distribution is 0.76 and the mode value is 0.85 . All three values are over 0.5 which indicates a good quality of the meshes. Another statistical study is 
convergence study of the solver we applied to solve CFD simulations. The solver applied is called a stationary solver which is used to solve steady-state problems. More precisely, Newton nonlinear method was applied in $\mathrm{COMSOL}^{\circledR}$. In this method, the iterative solver iterates until a relative tolerance is fulfilled. The tolerance was set to be 0.0001 in the solver.

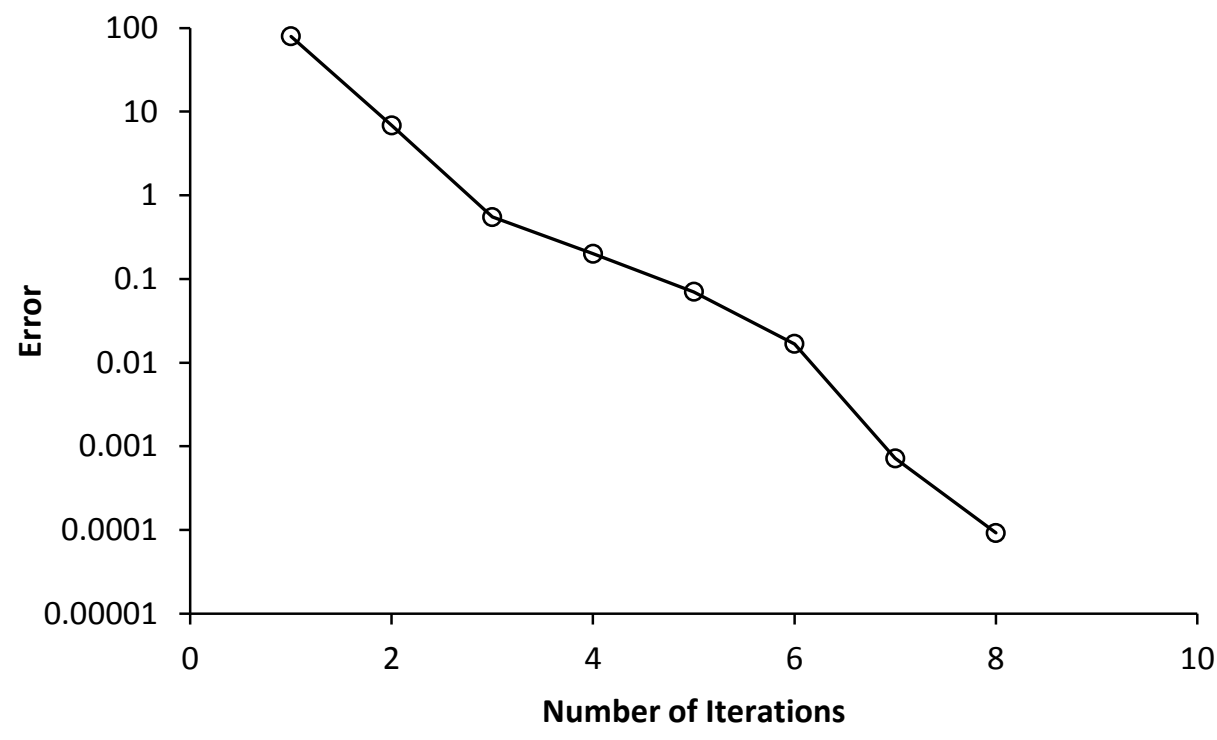

Figure 4. Relative error versus the iteration number of Newton nonlinear solver

Figure 4 indicates the relative error versus the iteration number. It can be seen from figure 4 that after 8 iterations, the relative error was smaller than the set tolerance (0.0001). In other words, the results were converged. These two statistical studies validated that the computational results are numerically correct with the same boundary conditions and similar meshes.

Ravensbergen et al. (Ravensbergen et al., 1995; Ravensbergen et al., 1997) measured the velocity distributions for the junction made of rectangular tubes as described above. Figure 5 shows the comparison between the CFD simulation and their experimental measurement. 
Figure 5. Simulation results (lines) and experimental data (discrete symbols) (Ravensbergen et al., 1995) comparisons for branching angle $63^{\circ}$ in (a) yz plane and (b) xz plane.

The continuous lines in the figure are simulation results while the discrete symbols are corresponding experimental measurement taken from (Ravensbergen et al., 1995). All the velocities are scaled by the mean axial velocity in the $z$ direction. Figure 5(a) shows the velocity profiles in the $y-z$ central plane while Figure 5(b) shows the velocity profiles in the $X-Z$ central plane. The values shown in Figure 5(b) indicate the distances (mm) in the z-direction measured from the branching point. It can be observed from the figure that the numerical and experimental results agree almost perfectly with each other. The maximum error is around 5\%. The CFD model can therefore be accepted as accurate enough for the branch design.

\section{Hemodynamic analysis for different branch designs}

CFD simulations are carried out for branches of different designs outlined at the beginning of section

2. The purpose of the analysis is to compare the different designs in terms of the wall shear stress (WSS) and flow behavior. As explained in the Introduction, WSS is the most important hemodynamic factor when designing a vascular network. Local recirculation should also be minimized to avoid nutrients (for arteries) or waste (for veins) to be trapped in a junction. The CFD results are presented in this section firstly for large vein vessels and then for micro-vascular vessels.

\subsection{CFD analysis for large vein branches}

CFD simulations were performed for cases 1, 2, and 5. In all cases two flows merge from the daughter vessels into the branch leading to a volume expansion. Using the same branching volume for all the junctions (continuous lines) in the $x z$ and $y z$ directions, respectively. 


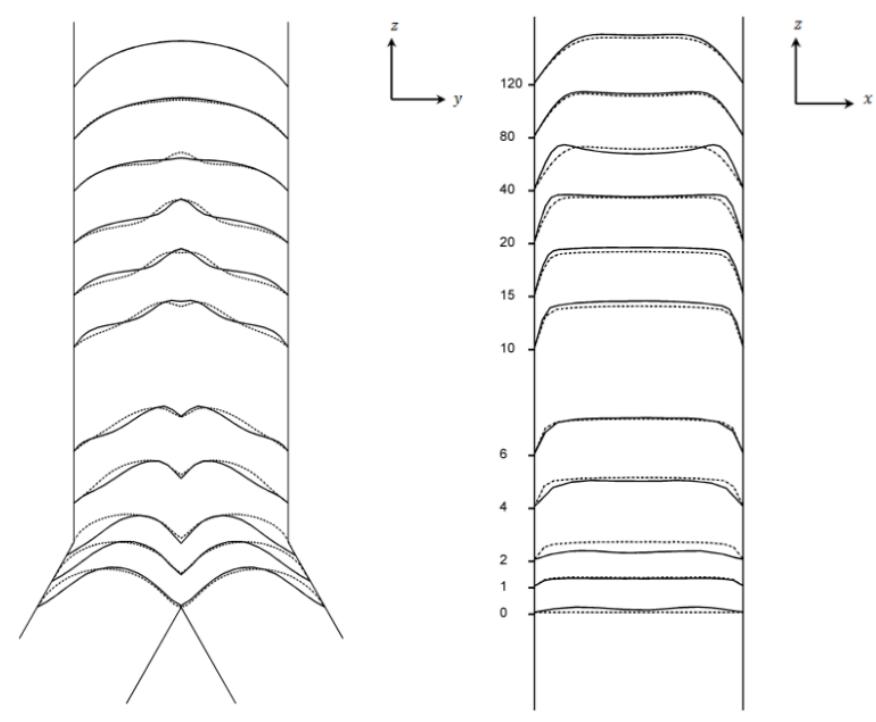

(a) $45^{\circ}$ : Case 5

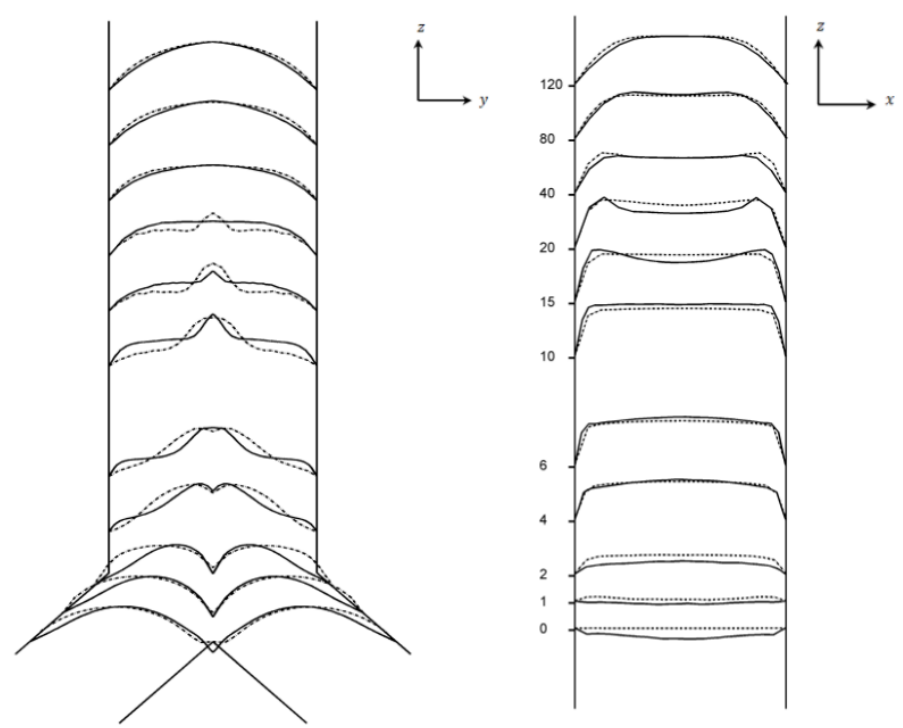

(b) $85^{\circ}$ : Case 2 

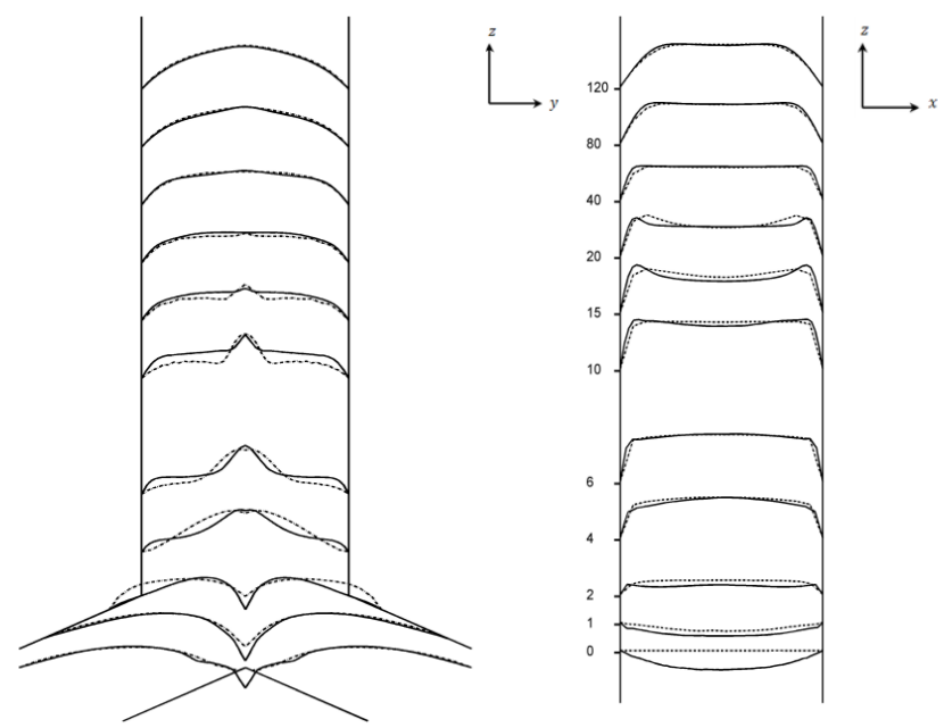

(c) $125^{\circ}$ : Case 1

203 tends to fully develop quicker than that of the sharp one while the peak profile disappears earlier. At 217 the end of the outlet, the two flow profiles are nearly identical. Similar trends are observed in cases 2, 2185 and their corresponding sharp counterparts. Three trends can be concluded by observing Figure 6: 1)

Figure 6. Comparisons between simulated velocity profiles in sharp branching junction (interrupted lines) and smoothed junctions (continuous lines) for angle (a) $45^{\circ}$ (b) $85^{\circ}$ (c) $125^{\circ}$ in both yz plane and $x z$ planes.

The branching angles of $45^{\circ}, 85^{\circ}$ and $125^{\circ}$ were used in these simulations. The interrupted lines and continuous lines at the bottom of in Figures 6(a-c) in $x z$ and $y z$ directions, compare the velocity profiles at the confluence positions for the sharp junctions and the same positions for the smoothed junctions, respectively. Two humps can be observed in both case 1 and the corresponding sharp junction in the area of influence. In case 1 , some negative velocities are observed, the values of which are larger than their sharp counterparts indicating a larger backflow. The two humps begin to merge downstream and start to generate peaks after $z=6 \mathrm{~mm}$ for both models. However, the peaks in the smoothed branch are narrower compared to that in the sharp branch. The flow becomes uniform further downstream for both smoothed and sharp branches. The flow profile of the smoothed model 
219 the magnitude of the negative flow at the branching point is larger for larger branching angle; 2) the

220 velocity profiles become smooth faster in the smoothed junctions compared with the sharp junctions;

221 3) the velocity profiles from rounded junctions approach those in the sharp junctions quicker for larger

222 branching angles. This also indicates that the branching angle has less influence in the smoothed

223 junctions.

224 To further demonstrate trend one in a two dimensional manner, two dimensional velocity 225 distribution around the axial centerline are presented in Figure 7 for branching angles of $45^{\circ}, 85^{\circ}$ and $226125^{\circ}$.

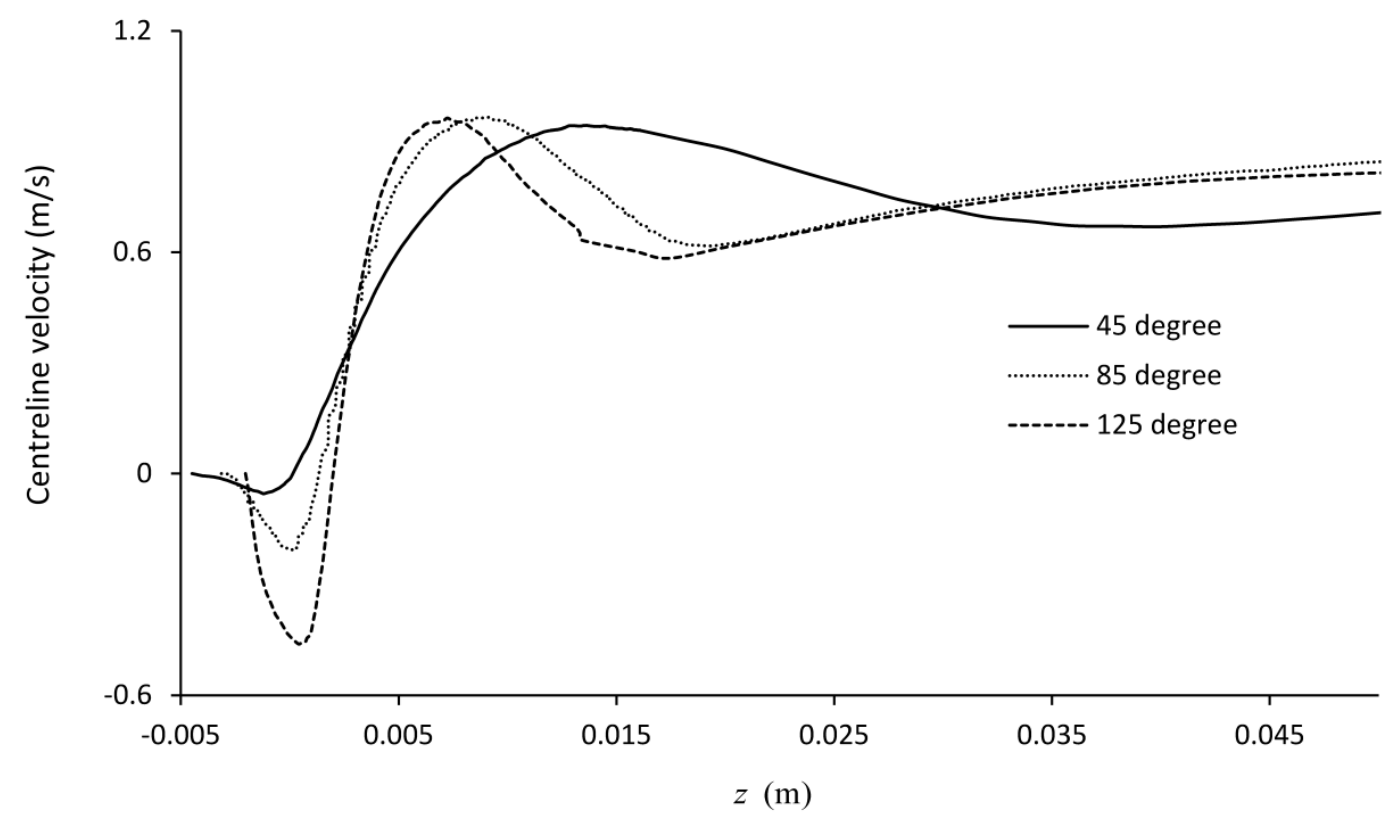

227

Figure 7. Centreline velocities for branching angles $45^{\circ}$ (case 5), $85^{\circ}$ (case 2) and $125^{\circ}$ (case 1 ).

229 Negative velocities can be observed in the branching areas for all the three smoothed junctions. A 230 junction with a larger branching angle has a larger value of negative velocity. The larger the branching 231 angles, the more it increases in the z-direction. At the range of $z=\sim 0.006$ to $0.015 \mathrm{~m}$, all the 232 velocities reach their peak values. The junctions with larger bunching angles reach their peak values 


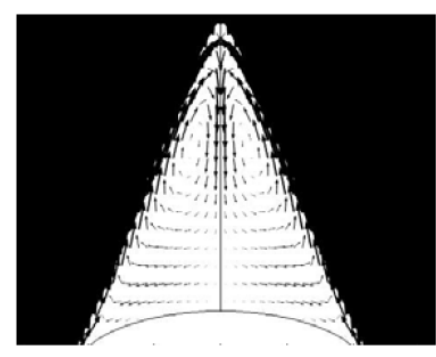

$45^{\circ}$

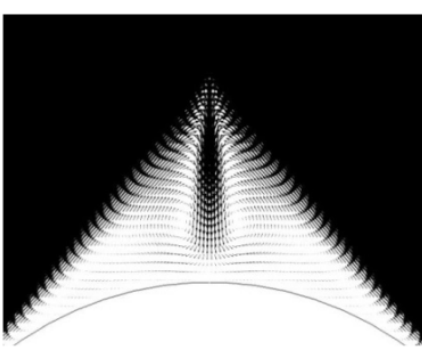

$85^{\circ}$

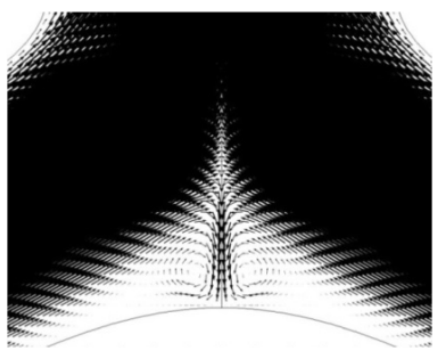

$125^{\circ}$
237

238

239

240

241

242

243

Figure 8. Recirculation area for branching angles $45^{\circ}$ (case 5), $85^{\circ}$ (case 2) and $125^{\circ}$ (case 1).

Two vortices can be seen in the branching area although its magnitude is small comparing with the surrounding velocity field. A region with low velocity vortices is known as a flow recirculation area.

Nutrients for arteries or waste for veins in the blood flow can be trapped in such area. Therefore, it is important to understand how the recirculation area of a rounded junction affects the flow velocity profile and the wall shear stress (WSS) downstream. In figure 8, the ratios of recirculation area over the whole branch are 1) $45^{\circ}: 26.4 \%$, 2) $85^{\circ}: 24.9 \%$ and 3) $125^{\circ}: 21.8 \%$. Junction with $45^{\circ}$ branch angle has the largest recirculation area while junction with $125^{\circ}$ branch angle has the smallest recirculation area. Although the junction at $125^{\circ}$ has the largest backflow, its recirculation area has the least influence on the velocity profile downstream. In order to explain this, the secondary velocity distribution for different cases at $z=6 \mathrm{~mm}$ is shown in Figure 9 (a) while those for branching angle of $125^{\circ}$ are shown in Figure 9 (b) at the positions of $z=6 \mathrm{~mm}, z=8 \mathrm{~mm}$ and $z=10 \mathrm{~mm}$. 


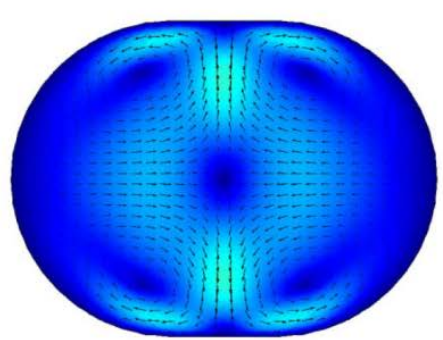

$45^{\circ}$

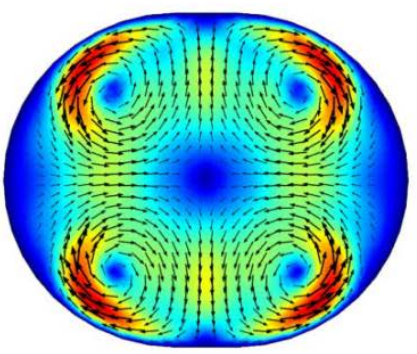

$z=6 \mathrm{~mm}$

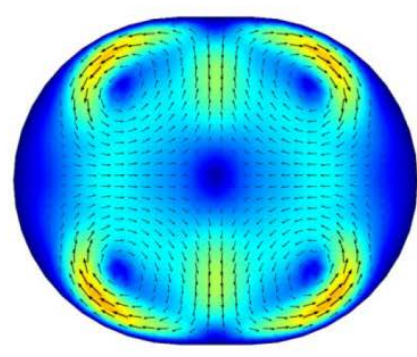

$85^{\circ}$

(a)

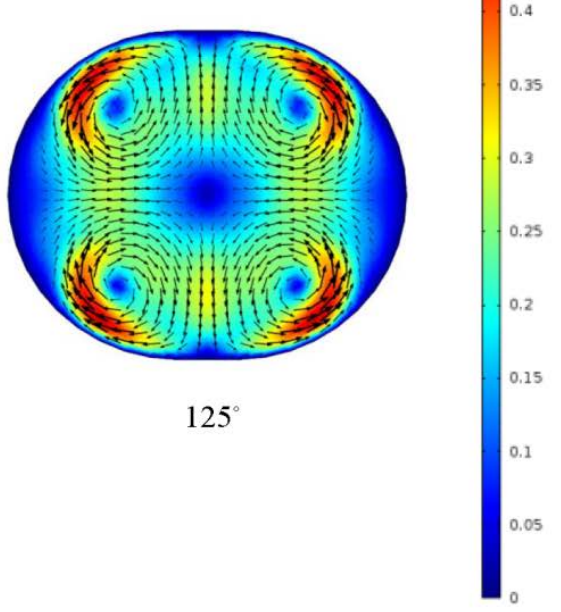

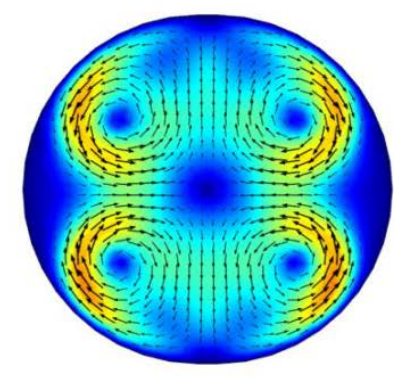

$z=8 \mathrm{~mm}$

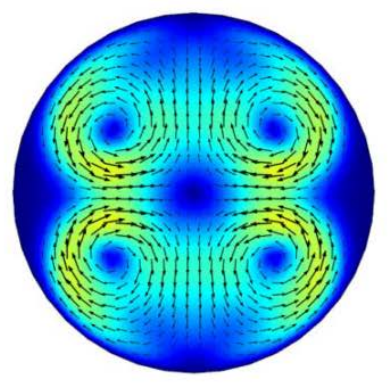

$z=10 \mathrm{~mm}$

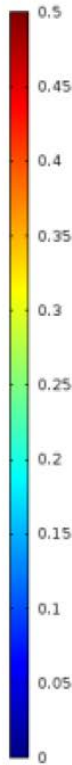

Figure 9. Secondary velocities for (a) branching angles $45^{\circ}, 85^{\circ}$ and $125^{\circ}$ at $z=6 \mathrm{~mm}$ and (b) branching angle $125^{\circ}$ at $z=6 \mathrm{~mm}, z=8 \mathrm{~mm}$ and $z=10 \mathrm{~mm}$

The vectors and magnitudes of the secondary velocity are presented in Figure 9 using colour plots for the three models. Four vortices can be found in each cross section. Figure 9 (a) illustrates a larger magnitude of the secondary velocity in the case of larger branching angle. A strong secondary vortex helps the two flows from the daughter vessels to mix well, which explain why in Figure 7 the flow profiles develop quicker in the cases of larger branching angles. A strong secondary vortex in a 

smoothed branching junction with a large branching angle diminishes the influence of the backflow.

261 This explains that even the backflow at the branching point of a larger angle junction is faster; its 262 influence on the velocity profile downstream is weaker. Hence, the main factor that influences the 263 downstream velocity profile is the size of the recirculation area rather than the strength of the backflow. Figure 9 (b) presents secondary velocities for smoothed junctions with a confluence angle of $125^{\circ}$ at different downstream positions of $z=6 \mathrm{~mm}, z=8 \mathrm{~mm}$ and $z=10 \mathrm{~mm}$. It is obvious that the

266 strength of the vortices decreases as $z$ increases. The vortices disappear once the flow is fully 267 developed.

268 The WSS is one of the most significant hemodynamic factors that relate to blood vessel development and cardiovascular diseases (Kohler et al., 2001; Caro, 2008; Coppola and Caro, 2008). In healthy cerebral arteries, the WSS ranges from 1 Pa to 7 Pa (Papaioannou and Stefanadis, 2004).

271 WSS higher than 7 Pa can damage the endothelial cells during vascular remodeling while WSS lower

272 than $1 \mathrm{~Pa}$ can lead to the formation of plaque due to insufficient mechanical stimulation on endothelial cells (Papaioannou and Stefanadis, 2004). WSS distributions for different smoothed cases and their sharp counterparts are shown in Figure 10. 

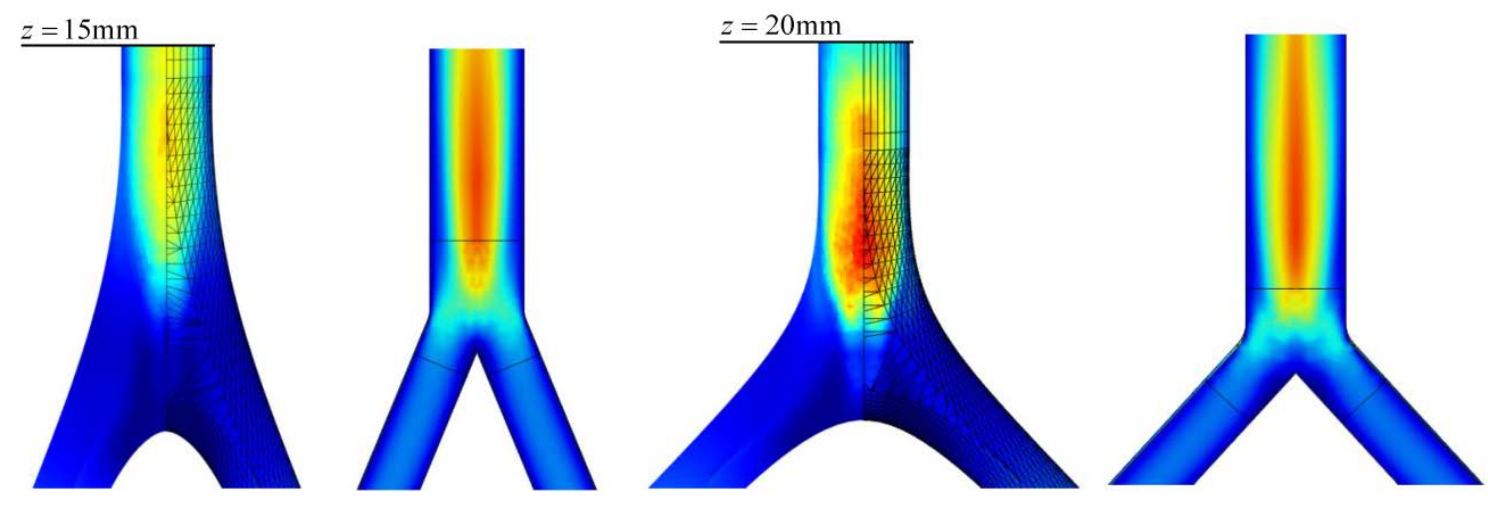

(a) $45^{\circ}$

(b) $85^{\circ}$

(pa)
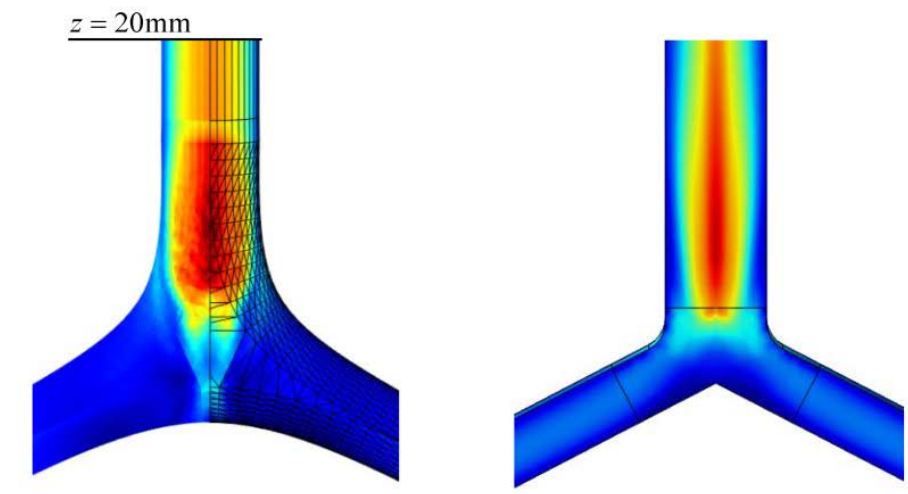

(c) 125

Figure 10. Wall shear stress distribution for branching angles (a) $45^{\circ}$, (b) $85^{\circ}$ and (c) $125^{\circ}$ for the smoothed model (left) and sharp model (right).

In the junction, the WSS can be many times higher than that in the straight vessel. WSS in the (12 Pa) is found on the sharp junction compared to the rounded one (10 Pa) as shown in Figure 10 (a).

The area of low WSS in the smoothed junction is larger than that in the sharp one due to recirculation.

Figure 10 (a) shows that at $z=15 \mathrm{~mm}$, the WSS distribution is more uniform with a low average value of $4 \mathrm{~Pa}$ in comparison with $5 \mathrm{~Pa}$ in a sharp junction. In Figure 10 (b), similar values of maximum WSS can be observed for both models ( 14 Pa). In the smoothed model, the distribution of WSS is more intense at the beginning of the downstream flow, but a more uniform distribution of low values is 
288 found at $z=20 \mathrm{~mm}$ in comparison with the sharp model. It is found in Figure 10 (c) that the 289 recirculation area has a similar but weak influence on the WSS distribution in a rounded junction 290 comparing those observed in a sharp junction for a confluence angle of $125^{\circ}$ shown. In order to 291 quantify how much the recirculation affects the WSS for different branching angles, a two 292 dimensional plot of WSS on a surface centerline is presented in Figure 11. 


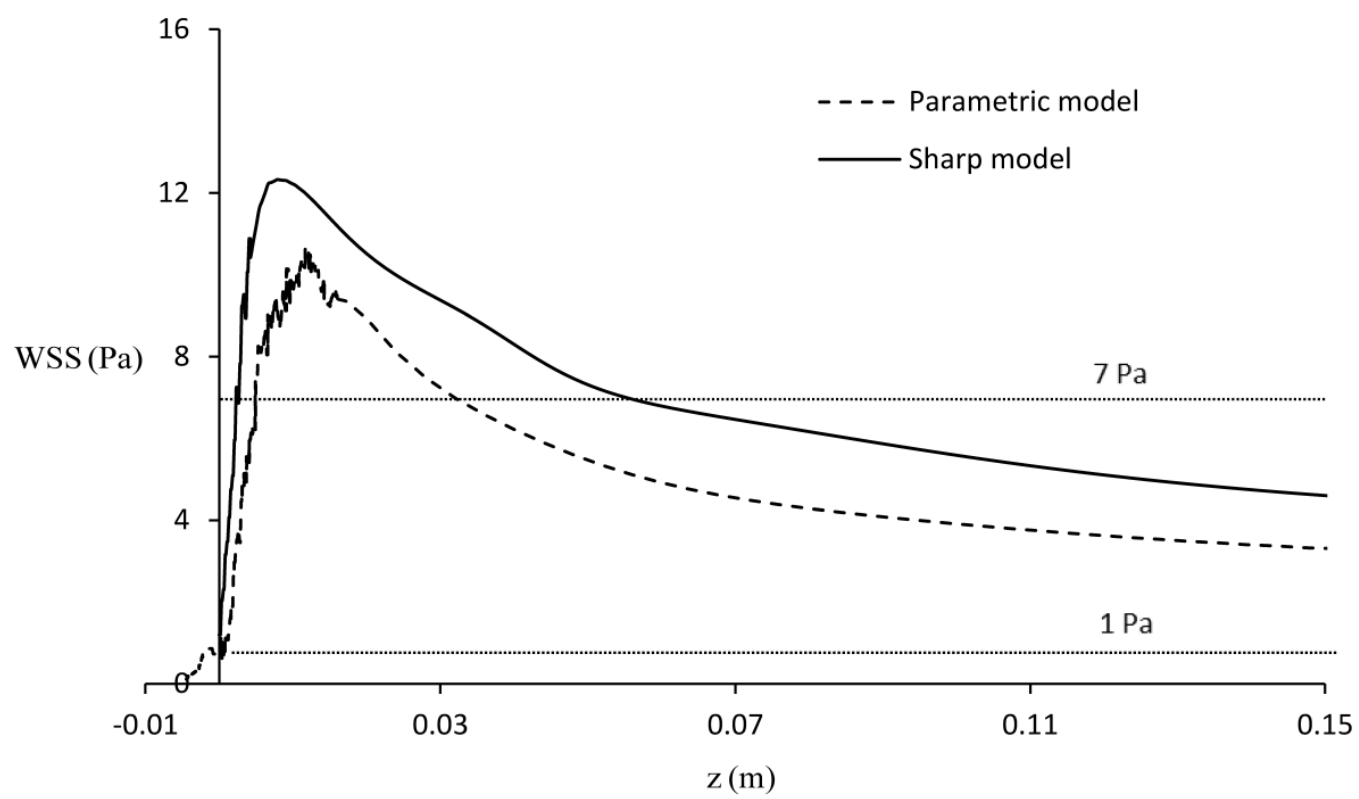

(a) $45^{\circ}$

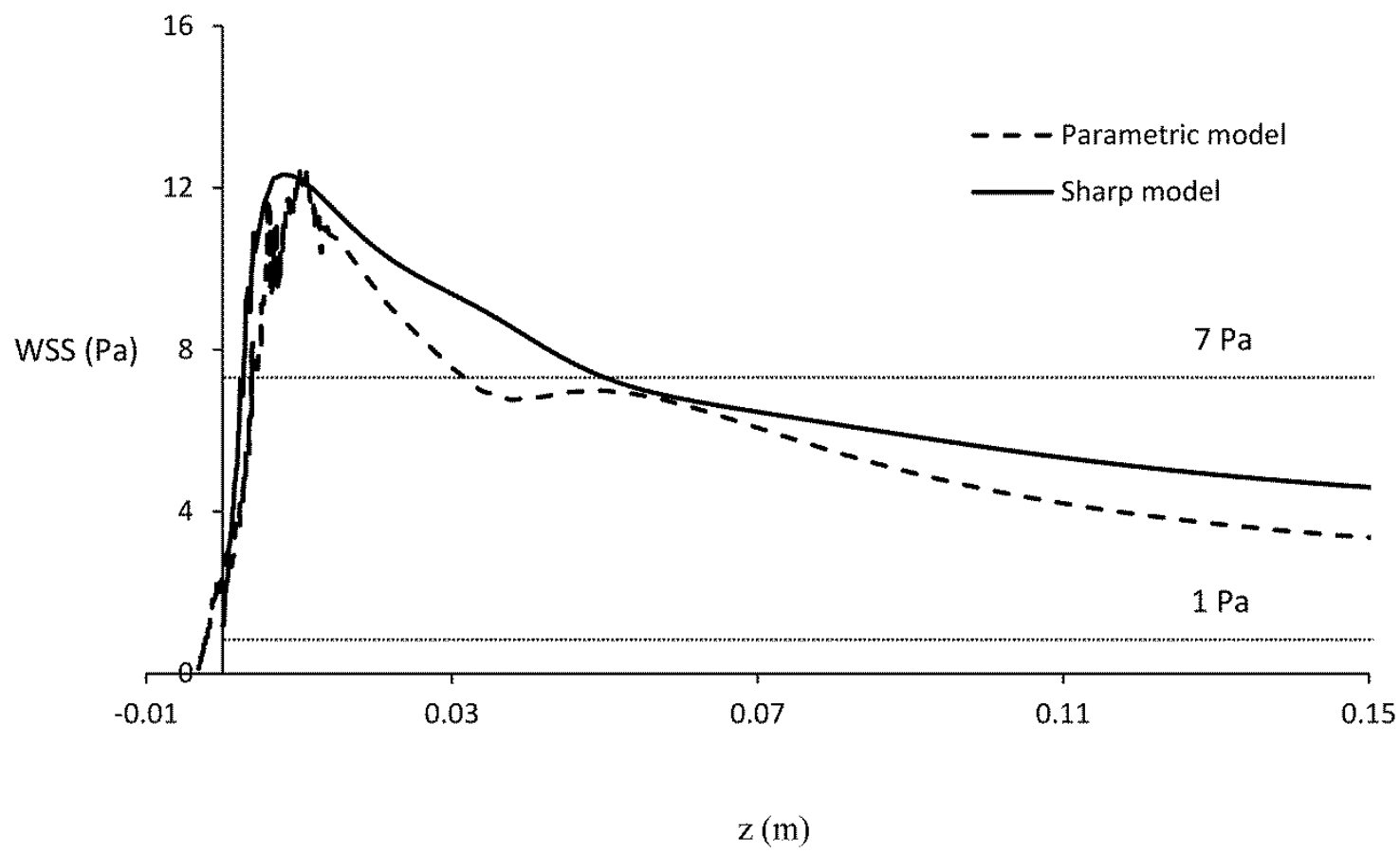

(b) $85^{\circ}$ 


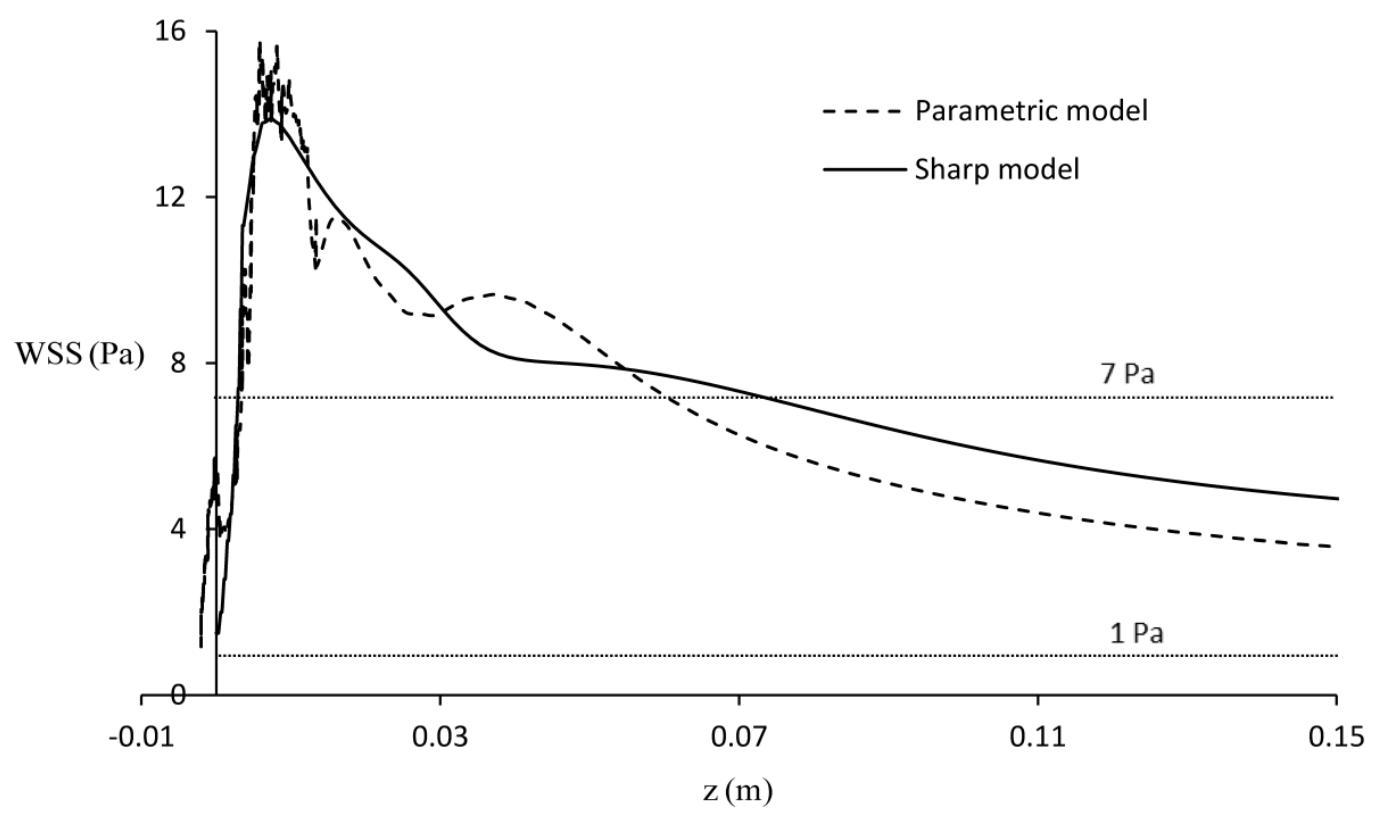

(c) $125^{\circ}$

296

297

298

299

300

301

302

303

304

305

306

307

Figure 11. WSS at the surface center line versus $\mathrm{z}$ of parametric model and sharp model, respectively for branching angles (a) $45^{\circ}$, (b) $85^{\circ}$ and (c) $125^{\circ}$.

The dashed lines and solid lines in Figure 11 show the WSS as a function of $\mathrm{z}$ for the smoothed and sharp junctions respectively for different branching angles of (a) $45^{\circ}$, (b) $85^{\circ}$ and (c) $125^{\circ}$. Here, a 'healthy window' is defined as the area along z-direction within which the WSS is in the range of $1 \mathrm{~Pa}$ to $7 \mathrm{~Pa}$. The healthy window is an important factor to assess a design of a junction. In the figure, an area with healthy WSS is indicated using two dashed lines, representing $1 \mathrm{~Pa}$ and $7 \mathrm{~Pa}$, respectively. In Figure 11 (a), the maximum WSS of the smoothed junction is smaller than that of the sharp one. The WSS values decrease for both junctions downstream. The healthy windows are $z \subset[0 \mathrm{~m}, 0.005 \mathrm{~m}]$ and $z \geq 0.06 \mathrm{~m}$ for the sharp junction as seen in Figure 11(a). Those windows expanded to $z \subset[0 \mathrm{~m}, 0.01 \mathrm{~m}]$ and $z \geq 0.035 \mathrm{~m}$ for the smoothed junction from as seen in Figure 11(a). The smoothed design of the junction improves the WSS distribution with wider healthy windows. Healthy window expansion can also be observed in Figures 11(b) and 11(c). All the smoothed junctions show a reduced 
311 The reduced amount is however different for the three branching angles. A correlation between the WSS reduction and the bifurcation angle is shown in figure 12.

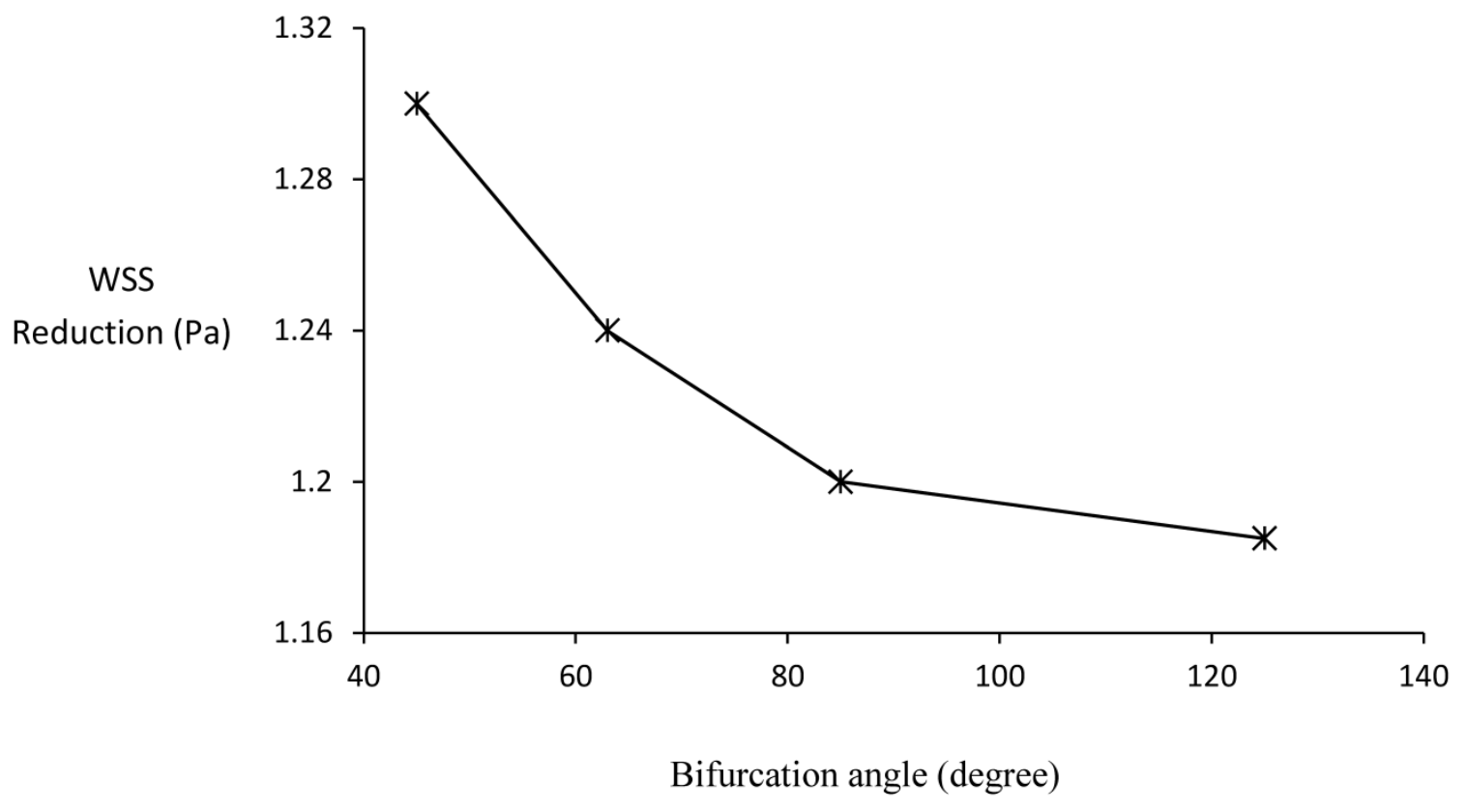

Figure 12. WSS reduction at $\mathrm{z}=0.15$ (m) using parametric model compared with sharp bifurcations versus bifurcation angles.

From Figure 12, it is seen that junctions with larger bifurcation angle results in a smaller WSS reduction. Further increase the bifurcation angle, the WSS reduction decreases more slowly. This indicates that the smoothed design has less effect on WSS reduction downstream for bifurcations with larger angle.

In the parametric design model of a branch junction, $\bar{C}_{\max }$ and its corresponding $\bar{V}$ are the most 322 important geometric parameters. A larger $\bar{C}_{\text {max }}$ leads to a smaller $\bar{V}$, thus a smaller branching area. 323 Further increasing $\bar{C}_{\text {max }}$, however, has a limited effect on the branching area as $\bar{V}$ will decrease more 324 slowly. In this section, CFD simulations are presented for smoothed junctions with different $\bar{C}_{\max }$ for a branching angle of $45^{\circ}$ (cases 3 - 7). Figure 13(a) shows the central axial velocity as a function of $z$ while Figure 13(b) shows the WSS along the centerline as a function of $\mathrm{z}$ coordinate for those cases. 


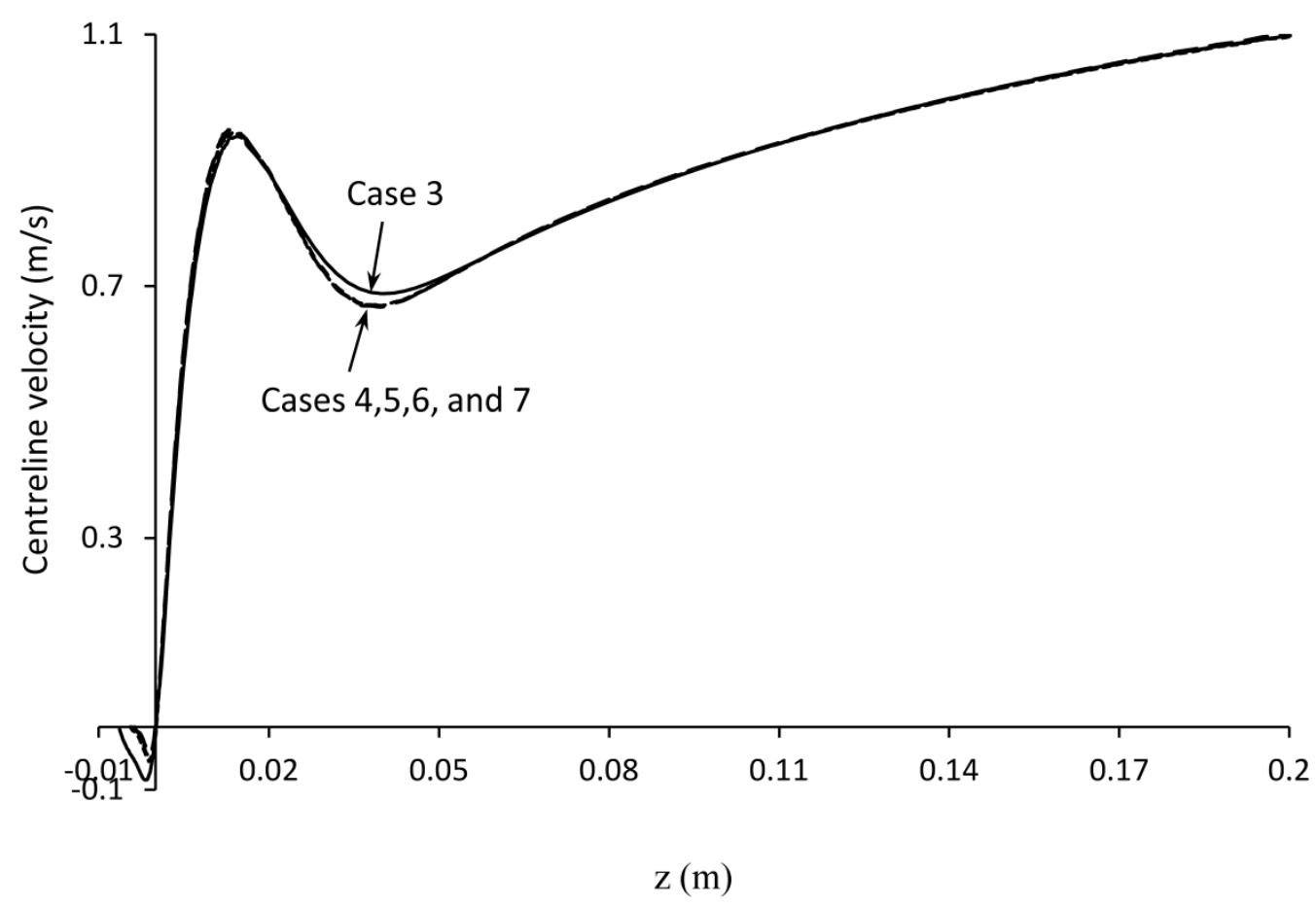

(a)

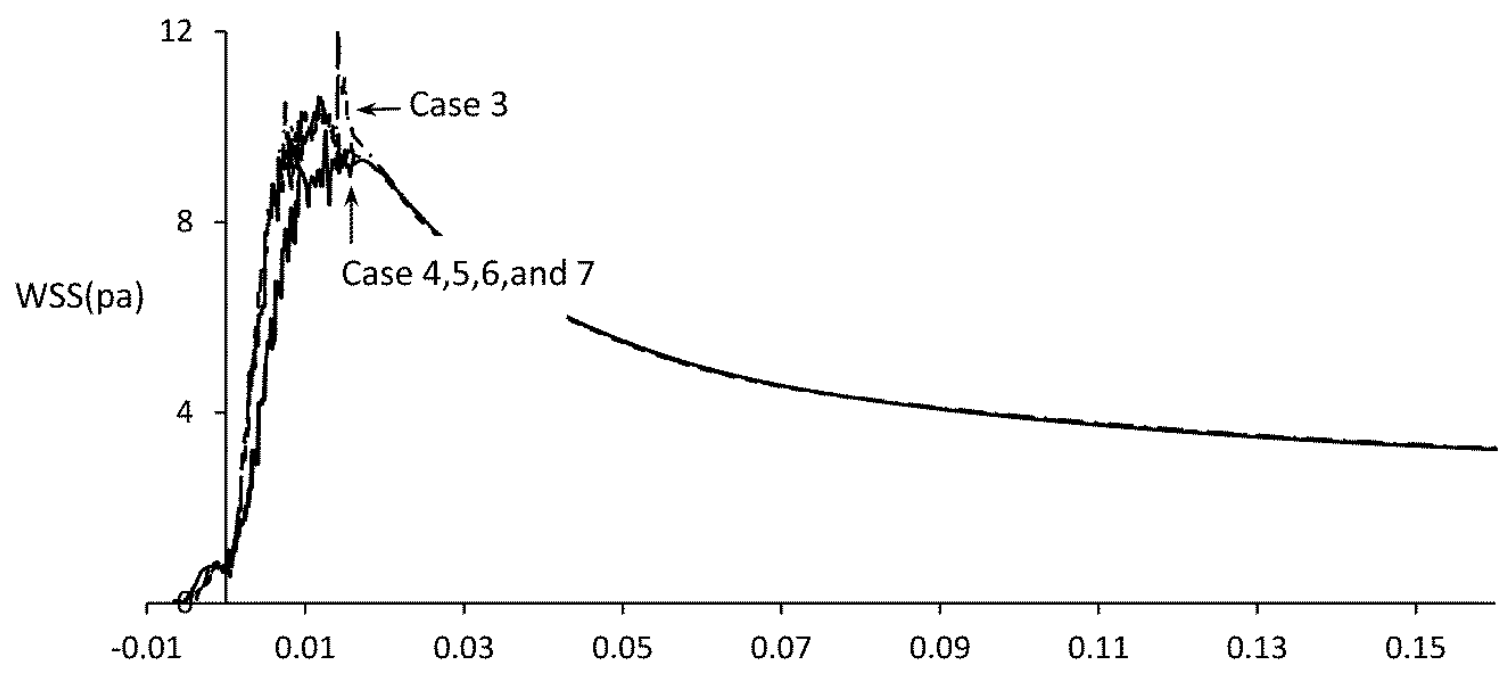

(b)

329 Figure 13. (a) Central axial velocities along center line for cases 3-7. (b) WSS at the surface center line versus $\mathrm{z}$ for cases 3-7.

332 In Figure 13(a), cases 4, 5, 6, and 7 show nearly identical velocity distributions. Only case 3 illustrates 333 small deviations from the other cases in the area of $z<0$ and $z \in(0.02 \mathrm{~m}, 0.05 \mathrm{~m})$. Figure 13(b) indicates 334 that WSS is different for the different junctions with different values of $\bar{C}_{\max }$ in the area of $\mathrm{z}<0.02 \mathrm{~m}$. 
335 Unsurprisingly case3 has the largest deviation. The WSS overlap with each other thereafter for all the 336 cases. It can be seen from table 2 that case 3 has the largest value of $\bar{V}$ and smallest value of $\bar{C}_{\max }$

337 while cases 4 to 7 have different values of $\bar{C}_{\max }$ but similar values of $\bar{V}$. This is why cases 4-7 have 338 similar central axial velocities and WSS. Although the velocities and WSS of case 3 show deviations 339 from the other cases, these deviations disappear downstream. These observations confirm that the 340 value of $\bar{C}_{\text {max }}$ and its corresponding $\bar{V}$ have effect on the distributions of central axial velocities and 341 WSS. The effects are, however, only in the local areas in contract to the influence from the branching 342 angle.

343 3.2. CFD analysis for micro-vascular vessels

344 Another important application of the parametric model is the design of micro-vascular networks for 345 supplying nutrients and oxygen in organ tissue engineering. In these micro-vascular networks, the 346 vessel diameters are in the range of tens of microns and the blood velocities are low which leads to a 347 small Reynolds number. In the simulations for the micro-vascular networks, all the diameters and inlet 348 velocities were scaled down by a factor of 0.01 from those in the previous section. The corresponding 349 Reynolds number is calculated as 0.06. Figure 14(a) compares the centerline velocities for cases with 350 branching angles of $45^{\circ}, 85^{\circ}$ and $125^{\circ}$ while Figure 14 (b) shows the WSS at the surface centerline as 351 functions of $\mathrm{z}$ for the same cases. 


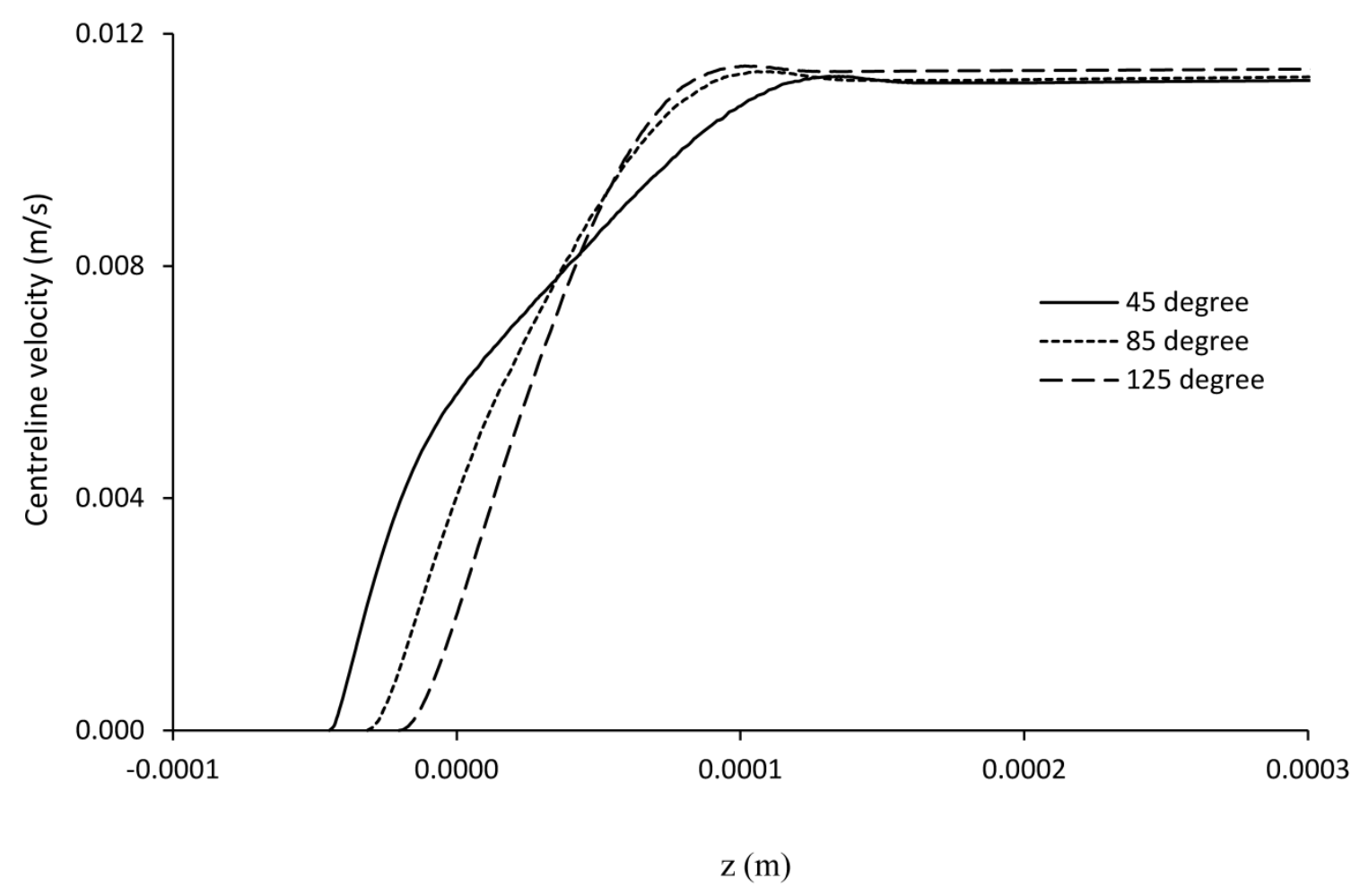

352

(a)

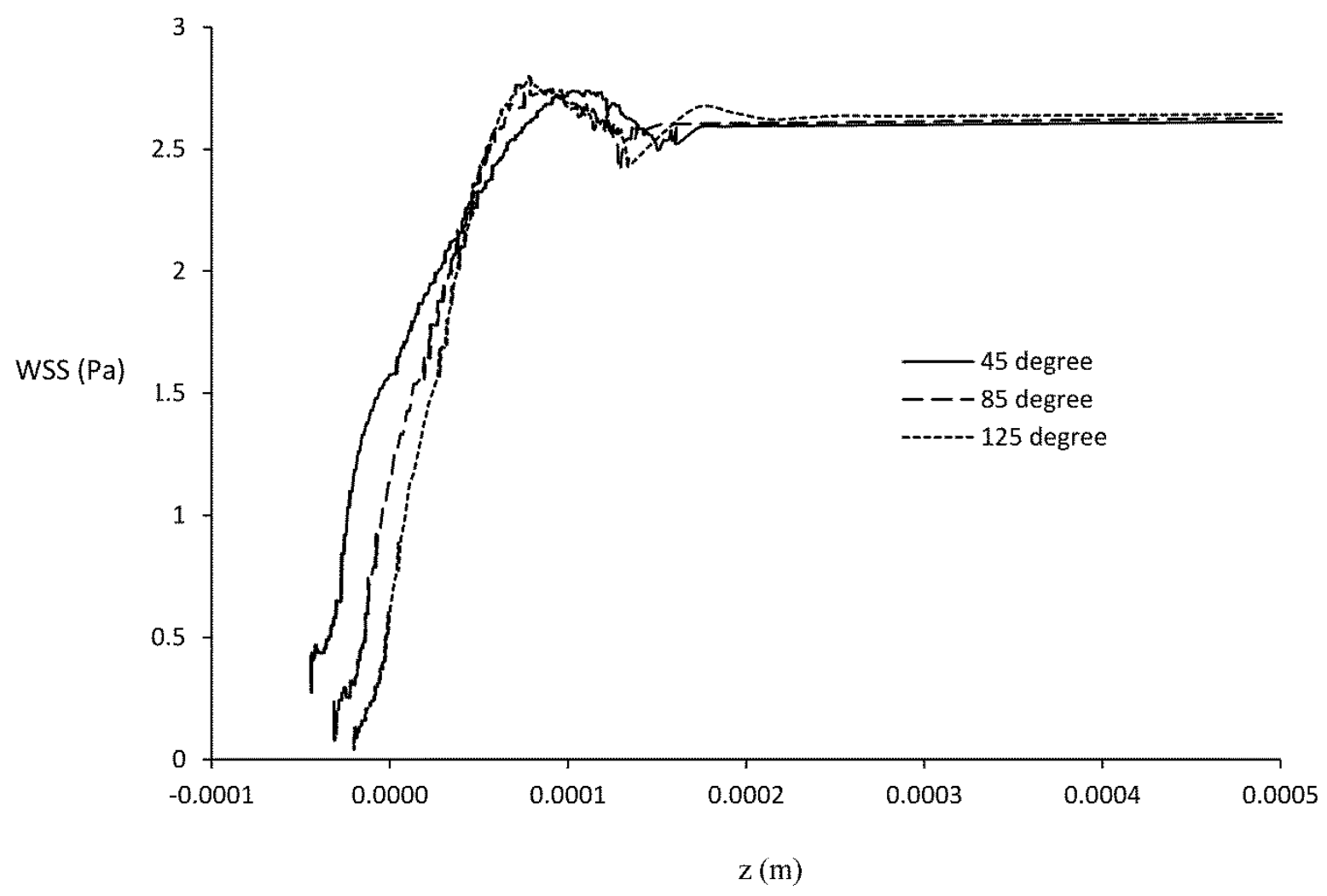


Figure 14. (a) Centerline velocities for branching angles $45^{\circ}, 85^{\circ}$ and $125^{\circ}$ and (b) WSS at the surface center line versus $\mathrm{z}$ of parametric model branching angles $45^{\circ}, 85^{\circ}$ and $125^{\circ}$, using a low Reynolds number.

It can be observed from figure 14(a) that the centerline velocities fully develop immediately after its maximum value regardless of the branching angles. Surprisingly, in the area where $\mathrm{z}$ is negative, no negative velocity can be observed for all the cases which means that backflow and flow recirculation do not exist. It can also be observed from Figure 14(b) that the WSS are only different in a small area of $\mathrm{z}<0.0002$ for all the cases of different branching angles.

\section{Conclusions}

In this paper, a set of CFD simulations were carried out to analyse the influence of the different geometric parameters in the branch design on flow behaviour. The geometric parameters include the branching angle, maximum curvature at the apex and volume of the branch. Firstly, a large Reynolds number of 600 was used in the simulations to represent arterial/venous flows. Then a small Reynolds number of 0.06 was applied to represent micro-vascular flows in a smaller lumen diameter. In the cases of large Reynolds number, it was found that in addition to manufacturing benefit, the smoothed junction has two advantages and one drawback. The advantages include: 1) it helps mixing the two flows better than the sharp junctions; and 2) they have large healthy windows of low WSS. The drawback is that the recirculation flow may occur in these smoothed junctions. The simulation results also revealed that different geometric parameters affect the flow behaviour differently: 1) a large branching angle tends to induce less recirculation flow and WSS reduction in smoothed junctions; and 2) the maximum curvature only affects the flow behaviour locally. For cases with low Reynolds number, the drawback does not exist because no recirculation was found in such cases. Therefore, designers are free to choose any $\bar{C}_{\max }$ for desired branch angles without considering the effect of the smoothed apex.

\section{Acknowledgements}

This work is part of the project ArtiVasc 3D (http://www.artivasc.eu/). It is financially supported by the European Union's Seventh Framework Programme (FP/2007-2013) under grant agreement No. 263416 (ArtiVasc 3D). 
383 Conflict of interest statement

384 We have no conflicts of interest to report. 


\section{References}

Caro, C. G., 2008. Discovery of the role of wall shear in atherosclerosis. Arteriosclerosis, Thrombosis, and Vascular Biology. 29, 158-161.

Cliff, W. J., 1976. Blood vessels. Cambridge: Cambridge University Press.

Coppola, G.; Caro, C. G., 2008. Arterial geometry, flow pattern, wall shear and mass transport: potential physiological significance. Journal of The Royal Society Interface. 1-10 p. 2008.

Edelman, E. R., 1999. Vascular Tissue Engineering - Designer Arteries. Circulation Research. 85, 11151117.

Friedman, M. H. et. al., 1983. Arterial geometry affects hemodynamics: a potential risk factor for atherogenesis. Atherosclerosis. 46, 225-231.

Friedman, M. H.; Seed, W. A., 1993, Hemodynamic implications of a relation between coronary geometry and pathology: Atherosclerosis. 98, 193-199.

Gibson, I.; Rosen, D. W.; Stucker, B., 2010. Additive manufacturing technologies: Rapid prototyping to direct digital manufacturing. Springer 2010.

Han, X.; Bibb, R.; Harris, R., 2015. Design of bifurcation junctions in artificial vascular vessels additively manufactured for skin tissue engineering. Journal of Visual Languages and Computing. 28, 238-249.

Hoch, E.; Tovar, G.; Borchers, K., 2014. Bioprinting of artificial blood vessels: current approaches towards a demanding goal. European Journal of Cardio - Thoracic Surgery. 46, 767-778.

Kamel, R. A. et al., 2013. Tissue Engineering of Skin. Journal of the American College of Surgeons. 217, 533-555.

Kannan, R. Y. et al., 2005. The roles of tissue engineering and vascularisation in the development of micro-vascular networks: a review. Biomaterials. 26, 1857-1875.

Kohler, U. et al., 2001. MRI measurement of wall shear stress vectors in bifurcation models and comparison with CFD predictions. Journal of Magnetic resonance imaging. 14, 563-573.

Kolesky, D. et al., 2014, 3D Bioprinting of Vascularized, Heterogeneous Cell-Laden Tissue Constructs: Adv.Mater. 26, 3124-3130.

Kucukgul, C. et al., 2013 3D hybrid bioprinting of macrovascular structures. Procedia Engineering. 59, 183-192.

Liu, G.; Wu, J.; Ghista, D. N. H., Wenhua Kelvin K.L. Wong., 2015. Hemodynamic characterization of transient blood flow in right coronary arteries with varying curvature and side-branch bifurcation angles. Computers in Biology and Medicine. 64, 117-126.

Marshall, I. et al., 2004. MRI and CFD studies of pulsatile flow in healthy and stenosed carotid bifurcation models. Journal of Biomechanics. 37, 679-687.

Miller, J. S., Kelly. et al., 2012. Rapid casting of patterned vascular networks for perfusable engineered three-dimensional tissues. Nature Materials. 11.

Naito, Y. et al., 2011. Vascular tissue engineering: towards the next generation vascular grafts. Advanced Drug Delivery Reviews. 65, 312-323. 
423 Papaioannou, T. G.; Stefanadis, C., 2004. Vascular wall shear stress: basic principles and methods.

424 Hellenic Journal of Cardiology. 46, 9-15.

425 Patrick, JR, C. W., 2000. Adipose tissue engineering: The future of breast and soft tissue 426 reconstruction following tumor resection. Seminars in Surgical Oncology. 19: 302-311 p. 2000.

427 Rose, F., Oreffo, R., 2002. Bone Tissue Engineering: Hope vs Hype. Biochemical and Biophysical 428 Research Communications. 292, 1-7.

429 Rabinovitz, R. S. Levesque, M. J. Nerem, R. M. 1987. Effects of branching angle in the left main 430 coronary bifurcation: Circulation. 76: IV-387.

431 Ravensbergen, J. et al., 1995. Merging flows in an arterial confluence: the vertebra-basilar junction. 432 Journal of Fluid Mechnaics. 304: 119-141.

433 Ravensbergen, J. et al., 1997. The influence of the blunting of the apex on the flow in a Vertebro434 Basilar junction model. Journal of Biomechanical Engineering. 119, 195-205.

435 Wu, W.; Deconinck, A.; Lewis, J., 2011. Omnidirectional Printing of 3D Microvascular Networks: Adv. 436 Mater. 23, $\mathrm{H} 178-\mathrm{H} 183$. 